\title{
Doença de Paget da mama: experiência de um centro universitário
}

ARTIGO ORIGINAL

\section{Descritores}

Neoplasias da mama

Doença de Paget da mama

Sobrevida

Eczema

\section{Paget's disease of the breast: experience of a university center}

Magno Belém Cirqueira', Leonardo Ribeiro Soares², Marise Amaral Rebouças Moreira³, Victor Domingos Lisita Rosa ${ }^{4}$, Ruffo Freitas-Junior ${ }^{5}$

\section{RESUMO}

Objetivo: Determinar a prevalência de doença de Paget da mama (DPM) entre os casos de carcinomas ductais diagnosticados em um centro universitário, entre 2003 e 2007, descrever as características clínicas e analisar a sobrevida desses casos. Métodos: Estudo de coorte retrospectiva, por meio da revisão de prontuários médicos. Foi realizada análise de frequência para todas as variáveis e utilizada curva de Kaplan-Meier para a representação da sobrevida global. Resultados: De 278 casos de carcinomas ductais de mama, houve 14 casos de DPM, determinando prevalência de 5,0\%. Um caso foi excluído da análise por apresentar dados incompletos. A média de idade ao diagnóstico foi de $57,1( \pm 11,2)$ anos. Dos casos analisados, $11(84,6 \%)$ apresentavam tumor palpável, e 9 (69,3\%), lesão do complexo aréolo-papilar (CAP). Apenas um caso não foi submetido à mastectomia, por óbito durante quimioterapia neoadjuvante. Radioterapia foi realizada em 6 casos (46,2\%), quimioterapia, em 11 casos (84,6\%), e endocrinoterapia, em 6 casos (46,2\%). A imunoistoquímica identificou 5 casos $(38,5 \%)$ com expressão de receptores hormonais e 12 casos $(92,3 \%)$ com superexpressão de HER2. A sobrevida global das pacientes foi de $61,5( \pm 13,4)$ meses e não houve recidiva local após um tempo médio de seguimento de 75,8 meses. Conclusáo: Observou-se prevalência de DPM associada a carcinomas invasores com estádio clínico avançado, o que possivelmente ocasionou sobrevida global inferior à observada em estudos prévios para a região.

\section{ABSTRACT}

Objective: To determine the prevalence of Paget's disease of the breast (PD) among cases of ductal carcinomas diagnosed in a university hospital between 2003 and 2007; describe clinical characteristics and analyze the survival of these cases. Methods: Retrospective cohort study, by reviewing medical records. Frequency analysis was performed for all variables and used Kaplan-Meier curve for the representation of overall survival. Results: In 278 cases of breast ductal carcinoma, 14 cases were PD determining prevalence of 5.0\%. A case was excluded from analysis because of incomplete data. The mean age at diagnosis was 57.1 ( \pm 11.2$)$ years. Of the cases analyzed, 11 (84.6\%) had palpable tumor and 9 (69.3\%) presented lesion of the nipple-areola complex. Only one case was not submitted to mastectomy because she died during neoadjuvant chemotherapy. Radiotherapy was performed in six cases (46.2\%); chemotherapy in eleven cases (84.6\%); endocrinoterapia in six cases (46.2\%).

Programa de Mastologia da Universidade Federal de Goiás (UFG) - Goiânia (G0), Brasil.

${ }^{1}$ Hospital das Clínicas da UFG - Goiânia (GO), Brasil.

${ }^{2}$ Setor de Ginecologia e Obstetrícia da UFG - Goiânia (GO), Brasil.

${ }^{3}$ Faculdade de Medicina da UFG - Goiânia (GO), Brasil.

${ }^{4}$ Setor de Oncologia Clínica da Universidade de São Paulo (USP) - Ribeirão Preto (SP), Brasil.

${ }^{5}$ Programa de Mastologia da UFG; Hospital Araújo Jorge da Associação de Combate ao Câncer em Goiás (ACCG) - Goiânia (GO), Brasil. Endereço para correspondência: Ruffo Freitas-Junior - Programa de Mastologia do Hospital das Clínicas da Universidade Federal de Goiás -

$1^{a}$ Avenida, s/n - Setor Universitário - CEP: 74605-050 - Goiânia (G0), Brasil - E-mail: ruffojr@terra.com.br

Conflito de interesses: nada a declarar.

Recebido em: 13/07/2015. Aceito em: 27/08/2015 
Immunohistochemistry identified five cases (38.5\%) with expression of hormone receptors and twelve patients (92.3\%) with overexpression of HER2. The overall survival of patients was 61.5 ( \pm 13.4 ) months, and there was no local recurrence after a mean follow-up of 75.8 months. Conclusion: There was a prevalence of Paget's disease of the breast associated with invasive carcinomas with advanced clinical stages; which possibly resulted in overall survival rate lower than that observed in other studies for the region.

\section{Introdução}

A doença de Paget da mama (DPM) é caracterizada pela presença de células malignas do epitélio glandular na papila mamária (células de Paget), com manifestação clínica de eritema e lesōes descamativas ${ }^{1-3}$. A associação da DPM com carcinoma mamário foi descrita inicialmente em 1874 pelo cirurgiāo britânico James Paget, o qual relatou 15 casos de mulheres entre 40 e 60 anos com alteraçóes cutâneas do complexo aréolo-papilar (CAP) que precediam a neoplasia ${ }^{4}$.

Clinicamente, a presença de prurido, descarga papilar ou ardência local pode ser referida. Na evoluçáo do processo podem ocorrer inversão, espessamento, erosão ou ulceração do mamilo e extensão da lesão à pele do complexo areolar ${ }^{2,3}$. A doença tem um curso insidioso e frequentemente é unilateral, com raros casos de acometimento bilateral ${ }^{2,5}$. Ao diagnóstico, cerca de $50 \%$ dos casos apresentam nódulos mamários palpáveis ${ }^{1,6}$.

O diagnóstico precoce de DPM é fundamental para a diminuição da morbidade e para o aumento da sobrevida livre de doença, possibilitando uma abordagem terapêutica mais individualizada ${ }^{3}$. Como a doença de Paget (DP) cursa com lesão eczematosa, o principal diagnóstico diferencial é o eczema de CAP, diferindo desse, por ser unilateral, com prurido de menor intensidade e com resposta inadequada à corticoterapia ${ }^{3}$.

O objetivo deste estudo foi determinar a prevalência de DPM entre os casos de carcinomas ductais diagnosticados em um centro universitário, entre 2003 e 2007, descrever características clínicas e analisar a sobrevida desses casos.

\section{Metodologia}

Trata-se de um estudo quantitativo, de coorte retrospectiva, que incluiu mulheres portadoras de DP associada a carcinoma ductal mamário. Os casos foram inicialmente selecionados em um banco de dados específico, composto por todos os casos de carcinoma ductal de mama diagnosticados no Programa de Mastologia do Hospital das Clínicas da Universidade Federal de Goiás (PM/UFG) entre os anos de 2003 e 2007.

Foram incluídos no presente estudo os casos com diagnóstico anatomopatológico de DPM, independentemente da apresentação clínica. Em uma segunda etapa, foi realizada a coleta de informaçôes nos respectivos prontuários médicos por meio do preenchimento de formulário próprio, o qual revisa informaçôes referentes à apresentaçáo clínica da doença e às características diagnósticas e terapêuticas de cada caso. Os critérios de exclusão foram aqueles com dados perdidos ou incompletos. O estudo atual faz parte de uma linha de pesquisa desenvolvida pela Rede Goiana de Pesquisa em Mastologia, com aprovação pelo Comitê de Ética em Pesquisa do Serviço (protocolo no 175/09).

As informaçóes coletadas foram tabuladas e analisadas com auxílio do programa Microsoft Office Excel ${ }^{\circledR}$, versão 2007 (Microsoft, EUA). Para a classificação das alteraçôes mamográficas utilizou-se o Breast Imaging Reporting and Data System $\left(\right.$ BI-RADS $\left.^{\text {тм}}\right)$, versão 2013, do American College of Radiology ${ }^{7}$. O estadiamento clínico foi realizado de acordo com o sistema TNM do American Joint Committee of Cancers (AJCC). A subclassificação do fenótipo tumoral foi realizada conforme descrição de Cheang et al. (2009), e utilizaram-se os valores de Ki- $67 \geq 14 \%$ como referência para a caracterização dos tumores luminais ${ }^{8,9}$. Foram realizadas a análise de frequência para todas as variáveis e a análise de tendência central para a determinação da média de idade. A sobrevida das pacientes foi calculada em meses completos após o diagnóstico histopatológico, com coleta do status vital em 29 de maio de 2015. Foi utilizado o método de Kaplan-Meier para a representação da curva de sobrevida global, por intermédio do programa Action, versão 2.8 (Estatcamp, BRA).

\section{Resultados}

Dos 278 casos de carcinomas ductais analisados, foram identificados 14 casos de DPM, determinando prevalência de 5,0\%. Um caso foi excluído do estudo por apresentar dados incompletos no prontuário médico. A idade ao diagnóstico variou entre 30 e 69 anos, com média de 57,1 anos e desvio padrão de $\pm 11,2$ anos (Tabela 1 ).

Entre os 13 casos analisados, $11(84,6 \%)$ apresentavam tumor palpável, e 9 (69,3\%), alteraçóes no CAP, incluindo lesóes ulceradas, eritematosas e/ou descamativas (Tabela 1 e Figura 1). $\mathrm{Na}$ avaliação mamográfica, 11 casos $(84,6 \%)$ foram classificados como BI-RADS 4 ou 5 , dos quais 4 casos $(30,7 \%)$ apresentavam microcalcificaçóes pleomórficas. 
Tabela 1. Características clínicas dos casos de doença de Paget da mama diagnosticados entre 2003 e 2007 no Programa de Mastologia da Universidade Federal de Goiás

\begin{tabular}{lccc}
\hline Variável & Média \pm DP & n & $\%$ \\
Média de idade (anos) & $57,1 \pm 11,2$ & 13 & 100 \\
Tumor palpável & & 11 & 84,6 \\
Metástase axilar & & 06 & 46,2 \\
& I & 03 & 23,1 \\
Estadiamento clínico & II & 04 & 30,7 \\
& III & 03 & 23,1 \\
& IV & 03 & 23,1 \\
\hline
\end{tabular}

DP: desvio-padrão.

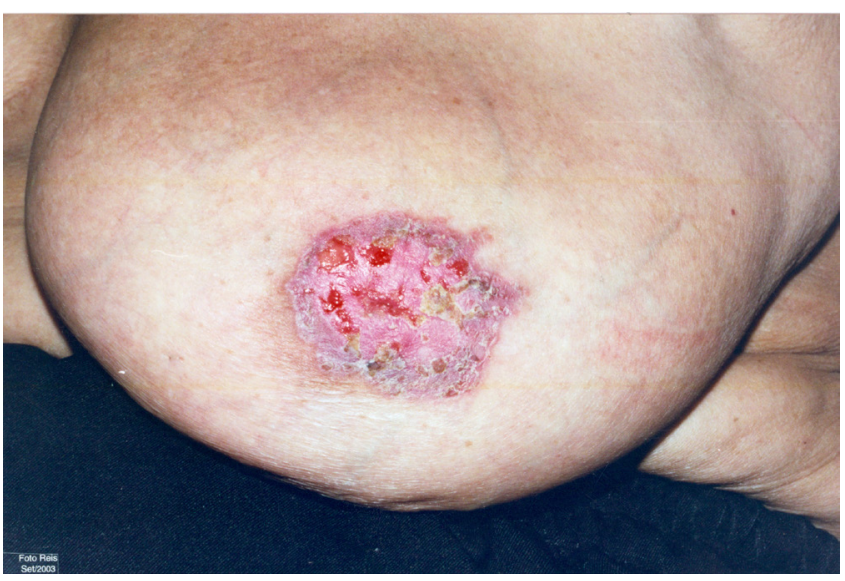

Figura 1. Lesão cutânea em mamilo esquerdo, pruriginosa, ulcerada, a qual apresentava bordas eritematosas e bem delimitadas
Apenas 1 caso $(7,7 \% \%)$ náo foi submetido à mastectomia, por óbito durante a quimioterapia neoadjuvante. Foi realizada reconstrução mamária em 9 casos (69,3\%), sendo utilizado retalho transverso do músculo reto abdominal (TRAM) em 7 casos e expansor de pele em 1 caso. Nas Tabelas 1 e 2 são observadas outras características diagnósticas e terapêuticas da amostra incluída no estudo.

As informaçóes anatomopatológicas dos casos descritos podem ser observadas na Tabela 3. A imunoistoquímica identificou 5 casos $(38,5 \%)$ com expressão de receptores hormonais e 12 casos $(92,3 \%)$ com superexpressão de HER2 (3+ ou FISH) (Tabela 2). Segundo a classificação fenotípica utilizada no Serviço ${ }^{8,9}$, houve prevalência dos subtipos superexpressão de HER2 (61,5\%) e luminal B (38,5\%).

A Figura 2 representa a curva de sobrevida global das pacientes incluídas no presente estudo, cuja média foi de $61,5( \pm 13,4)$ meses. Após um tempo médio de seguimento de 75,8 meses, duas pacientes perderam o seguimento clínico no Serviço e três pacientes evoluíram a óbito. Não foi observada recidiva locorregional no período analisado. Na Figura 3 podem ser observadas as características anatomopatológicas da DPM diagnosticada em um caso da presente série.

\section{Discussão}

A prevalência de DP associada a carcinoma mamário é baixa, variando entre 0,5 e $5,0 \%$ dos casos ${ }^{10-12}$. Na presente série encontrou-se prevalência de $5,0 \%$, possivelmente em decorrência de a amostra analisada ser proveniente de um hospital terciário,

Tabela 2. Série de casos de doença de Paget da mama diagnosticados entre 2003 e 2007 no Programa de Mastologia da Universidade Federal de Goiás

\begin{tabular}{|c|c|c|c|c|c|c|c|c|}
\hline \multirow{2}{*}{ Caso } & \multirow{2}{*}{ Idade } & \multirow{2}{*}{$\begin{array}{l}\text { Alteraçáo na } \\
\text { pele }\end{array}$} & \multirow{2}{*}{$\begin{array}{l}\text { Tumor } \\
\text { palpável }\end{array}$} & \multirow{2}{*}{ BIRADS $^{\mathrm{TM}}$} & \multirow{2}{*}{$\mathrm{EC}$} & \multicolumn{2}{|c|}{ Tratamento } & \multirow{2}{*}{ Status vita } \\
\hline & & & & & & Cirurgia & QT/RT/HT & \\
\hline 1 & 37 & Sim & Sim & 4 & IV & Mastectomia & QT-RT-HT & Vivo \\
\hline 2 & 30 & Sim & Não & 4 & I & Mastectomia & - & Vivo \\
\hline 3 & 69 & Sim & Sim & 4 & II & Mastectomia & QT & N/A \\
\hline 4 & 63 & Sim & Não & 5 & II & Mastectomia & QT-HT & Vivo \\
\hline 5 & 54 & Sim & Sim & 5 & III & Mastectomia & QT-RT-HT & Óbito \\
\hline 6 & 47 & Não & Sim & 5 & III & Mastectomia & QT-RT & Vivo \\
\hline 7 & 49 & Não & Sim & 4 & IV & Mastectomia & QT-RT & N/A \\
\hline 8 & 44 & Sim & Sim & 4 & I & Mastectomia & QT & Vivo \\
\hline 9 & 52 & Não & Sim & 5 & II & Mastectomia & HT & Vivo \\
\hline 10 & 57 & Sim & Sim & 5 & III & - & QT & Óbito \\
\hline 11 & 37 & Sim & Sim & 0 & IV & Mastectomia & QT-RT & Óbito \\
\hline 12 & 53 & Sim & Sim & 4 & II & Mastectomia & QT-HT & Vivo \\
\hline 13 & 37 & Não & Sim & 3 & I & Mastectomia & QT-RT-HT & Vivo \\
\hline
\end{tabular}

BIRADS $^{\mathrm{TM}}$ : Breast Imaging Reporting and Data System, 2013; EC: estadiamento clínico; QT: quimioterapia; HT: hormonioterapia; RT: radioterapia; N/A: não avaliado (perda de seguimento); idade: em anos. 
Tabela 3. Características anatomopatológicas de uma série de casos de doença de Paget da mama diagnosticados entre 2003 e 2007 no Programa de Mastologia da Universidade Federal de Goiás

\begin{tabular}{lccccc}
\hline Caso & Tipo & Grau & Receptores hormonais (RE/RP) & HER2 & Subtipo \\
1 & CDI & 2 & POS & POS & Luminal B \\
2 & CDis & - & NEG & POS & Superexpressão de HER2 \\
3 & CDI & 2 & NEG & POS & Superexpressão de HER2 \\
4 & CDI & 3 & POS & POS & Luminal B \\
5 & CDI & 2 & POS & POS & Luminal B \\
6 & CDI & - & NEG & POS & Superexpressão de HER2 \\
\hline 7 & CDI & 2 & NEG & POS & Superexpressão de HER2 \\
\hline 8 & CDI & 2 & NEG & POS & Superexpressão de HER2 \\
9 & CDis & - & NEG & POS & Superexpressão de HER2 \\
10 & CDI & 2 & NEG & POS & Superexpressão de HER2 \\
11 & CDI & 2 & NEG & POS & Superexpressão de HER2 \\
12 & CDI & 2 & POS & POS & Luminal B \\
13 & CDI & 2 & POS & NEG & Luminal B \\
\hline
\end{tabular}

CDI: carcinoma ductal invasor; CDis: carcinoma ductal “in situ”; Grau: grau histológico; RE: receptor de estrogênio, RP: receptor de progesterona; POS: positivo: NEG: negativo.

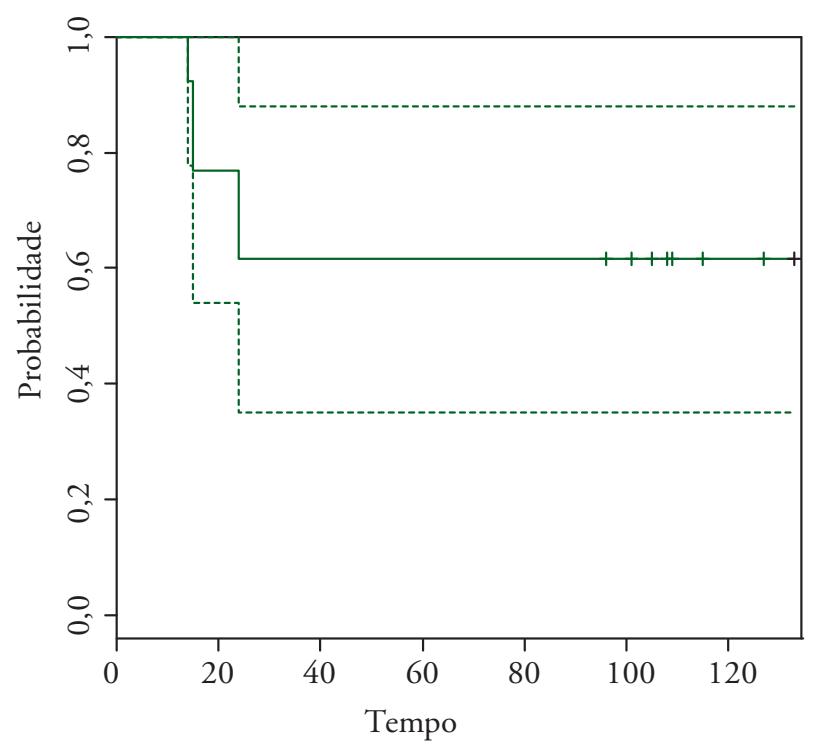

Figura 2. Curva de Kaplan-Meier mostrando a sobrevida global (em meses) dos casos de doença de Paget da mama diagnosticados no Programa de Mastologia da Universidade Federal de Goiás entre 2003 e 2007; probabilidade: proporção cumulativa de sobrevida global; tempo de sobrevida: em meses

de referência regional para o tratamento do câncer de mama, com admissão de pacientes portadoras de doença avançada e de maior complexidade ${ }^{13}$. Outro aspecto que talvez possa justificar essa prevalência é a inclusão de casos detectados pela anatomia patológica, porém sem a lesão cutânea clássica, o que ocorreu em 4 dos 13 casos incluídos. Apesar de parecer elevada, taxa ainda maior foi observada em outro estudo brasileiro, conduzido

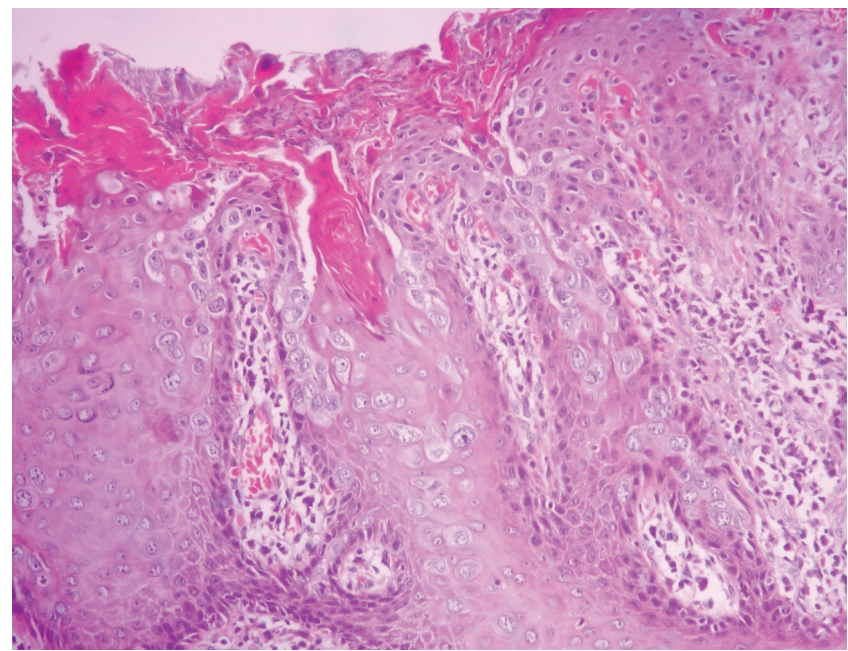

Figura 3. Microfotografia de mamilo (HE, 100x) evidenciando a presença de células arredondadas ou ovoides, com núcleo volumoso e hipercromático, nucléolo evidente e citoplasma abundante e claro (células de Paget)

na cidade de Campinas, São Paulo, no qual 46\% dos casos náo apresentavam alteraçóes do $\mathrm{CAP}^{14}$.

A presença de lesóes cutâneas poderia constituir um aspecto favorável ao diagnóstico precoce do carcinoma mamário associado à DP (Figura 2). No entanto, verificou-se atraso diagnóstico de até 18 meses na casuística de Campinas, São Paulo, provavelmente em decorrência do diagnóstico diferencial de eczema de $\mathrm{CAP}^{14}$. Em nossa série, a maioria das pacientes apresentava lesóes cutâneas ou nódulos palpáveis, porém o tempo entre o início dos sintomas e o diagnóstico de DPM não foi confirmado em decorrência da ausência dessa informação nos prontuários médicos. 
Entre os exames de imagem, a ultrassonografia mamária e a ressonância nuclear magnética (RNM) têm sido propostas como métodos de complementação diagnóstica, na avaliação de lesões mamográficas suspeitas e na detecção de lesôes ocultas à mamografia, em pacientes com diagnóstico de $\mathrm{DPM}^{6}$. Em nossa série, a mamografia revelou nódulos suspeitos e/ou microcalcificações pleomórficas na maioria dos casos e não houve nenhuma solicitação de RNM devido à ausência de disponibilidade do método no período estudado. No entanto, apesar das taxas de falso-negativos observados em estudos de avaliação da mamografia ${ }^{14,15}$, não houve prejuízo diagnóstico ou terapêutico pela não utilização da RNM na amostra descrita.

A imunoistoquímica pode ser importante para a confirmação diagnóstica de DPM (Figuras 4 e 5), a qual expressa os marcadores CK-7, AE1, AE3, CEA e EMA 3,16,17. As células de Paget são PAS-positivas e contêm mucina ácida, o que também colabora para o diagnóstico diferencial ${ }^{16}$. Ainda, deve-se destacar a baixa expressão de receptores de estrogênio ou progesterona ${ }^{18}$. Em nossa amostra apenas $38,5 \%$ das pacientes com DPM apresentavam positividade para receptores hormonais, o que justifica a taxa de endocrinoterapia $(46,2 \%)$ observada entre os casos incluídos.

A superexpressão de HER2 é reportada entre 80 e 100\% dos casos de DPM ${ }^{18,19}$ e normalmente está associada a tumores extensos, de alto grau e com pior prognóstico ${ }^{18-20}$. No entanto, a consolidação do Trastuzumabe e de outras terapias-alvo dirigidas tem aumentado a sobrevida global das pacientes portadoras de tumores mamários com superexpressão de HER $2^{21,22}$. No estudo atual, apesar da expressão positiva de HER2 em 92,3\% dos tumores, nenhuma paciente utilizou Trastuzumabe, devido a não disponibilidade da medicação no período analisado. Essa limitação terapêutica

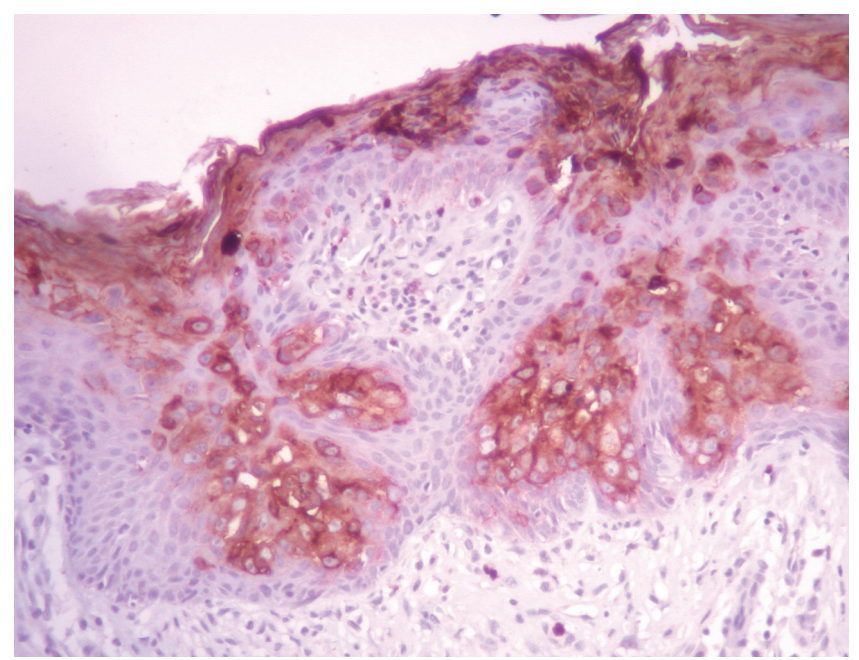

Figura 4. Microfotografia (100x) mostrando estudo imunoistoquímico de lesão em complexo aréolo-papilar e coloração positiva para CEA policlonal pode ter contribuído para a curva de sobrevida global observada na presente amostra, a qual foi inferior em relação aos demais estudos de sobrevida por câncer de mama realizados na cidade de Goiânia, Goiás ${ }^{23,24}$.

Em decorrência da baixa incidência de DPM, não existem estudos randomizados avaliando a melhor estratégia terapêutica para tal patologia. Historicamente, o tratamento cirúrgico recomendado é a mastectomia, considerando-se a possibilidade de lesōes mamárias extensas, multifocais ou multicêntricas ${ }^{10,25}$. Porém, têm sido observados resultados semelhantes entre a mastectomia e as técnicas cirúrgicas conservadoras na sobrevida global das pacientes ${ }^{26,27}$, embora a taxa de recorrência local seja significantemente maior entre as mulheres submetidas à cirurgia conservadora ${ }^{14}$. Estudos prospectivos e multicêntricos devem ser conduzidos para auxiliar o cirurgião acerca da abordagem cirúrgica mais adequada, visto que a descrição de novas técnicas de oncoplástica ${ }^{28} \mathrm{e}$ a evolução das mastectomias ${ }^{29}$ podem modificar as taxas de recidiva observadas atualmente. Na presente série, todas as pacientes foram submetidas à mastectomia por seguirmos o protocolo de tratamento de DPM do PM/UFG, embora o tratamento cirúrgico seja discutido e proposto individualmente para cada paciente.

Em um estudo clássico que incluiu 223 casos de DPM, a taxa acumulada de recorrência local foi de $9 \%$, sendo de $8 \%$ em pacientes tratadas com mastectomia e de $16 \%$ entre as pacientes tratadas com cirurgia conservadora da mama, sem diferença significativa na sobrevida global ou na sobrevida livre de doença entre os dois procedimentos ${ }^{2}$. Essa mesma situaçáo foi observada pelo grupo da Universidade Estadual de Campinas (UNICAMP), São Paulo ${ }^{14}$. Em nossa série, não foi observada recidiva locorregional após um tempo médio de 75,8 meses de seguimento, o que pode ser decorrente do predomínio de

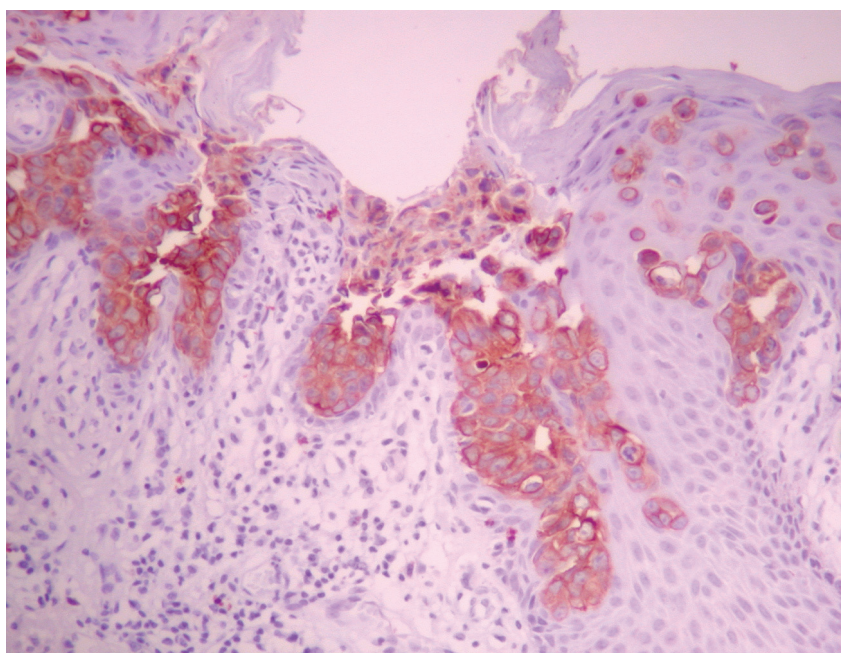

Figura 5. Microfotografia (100x) mostrando estudo imunoistoquímico de lesão em complexo aréolo-papilar e coloração positiva para CK7 
mastectomias na abordagem cirúrgica da doença ou do número reduzido de casos analisados.

Considerando os desfechos clínicos comumente utilizados em oncologia, as taxas de sobrevida global representam um importante parâmetro de avaliação de resultados. Entre as portadoras de DP e carcinoma mamário, a presença de lesões cutâneas no CAP se mostrou associada a formas menos agressivas da doença e a maior sobrevida global ${ }^{14}$. Em nossa série, foi observada uma elevada prevalência de fatores prognósticos desfavoráveis, como tumores palpáveis ao diagnóstico e comprometimento metastático axilar e/ou à distância; porém, o número restrito de casos limitou a associação entre essas variáveis e a curva de sobrevida das pacientes. Durante o seguimento clínico, foram observados 3 óbitos na população analisada e uma sobrevida global média de 61,5 meses, a qual está abaixo da sobrevida observada em outros estudos nacionais ${ }^{23,24,30}$, possivelmente em decorrência do estadiamento avançado ao diagnóstico.

\section{Conclusão}

Observou-se prevalência de DPM associada a carcinomas invasores com estádio clínico avançado, o que possivelmente ocasionou sobrevida global inferior à observada em estudos prévios para a região. Esses dados indicam a necessidade de maior difusão de informaçôes acerca das alteraçôes cutâneas observadas na DPM.

\section{Referências}

1. Sandoval-Leon AC, Drews-Elger K, Gomez-Fernandez CR, Yepes MM, Lippman ME. Paget's disease of the nipple. Breast Cancer Res Treat. 2013;141(1):1-12.

2. Dalberg K, Hellborg H, Wärnberg F. Paget's disease of the nipple in a population based cohort. Breast Cancer Research and Treatment. 2008;111(2):313-19.

3. Lage D, Volpini CA, Sasseron MG, Daldon P, Arruda L. Doença de Paget: a importância do especialista. An Bras Dermatol. 2010;85(3):365-9.

4. Paget J. On the disease of the mammary areola preceding cancer of the mammary gland. St Barth Hosp Rep. 1874;10:87-9.

5. Barth D. Bilateral Paget's disease of the breast-case report of longtime misdiagnosed tumors with underlying ductal carcinomas and review of the literature. Case Rep Dermatol Med. 2014;2014:152836.

6. Kawase K, Dimaio DJ, Tucker SL, Buchholz TA, Ross MI, Feig BW, et al. Paget's disease of the breast: there is a role for breast-conserving therapy. Ann Surg Oncol. 2005;12(5):391-7.

7. Mendelson EB, Bohm-Vélez M, Berg WA, et al. ACR BIRADS ${ }^{\circ}$ Ultrasound. In: ACR BI-RADS Atlas, Breast Imaging Reporting and Data System. Reston, VA: American College of Radiology; 2013.

8. Cheang MC, Chia SK, Voduc D, Gao D, Leung S, Snider J, et al. Ki67 index, HER2 status, and prognosis of patients with luminal B breast cancer. J Natl Cancer Inst. 2009;101(10):736-50.
9. Goldhirsch A, Wood WC, Coates AS, Gelber RD, Thürlimann B, Senn H-J, et al. Strategies for subtypes - dealing with the diversity of breast cancer: highlights of the St Gallen International Expert Consensus on the primary therapy of early breast cancer 2011. Ann Oncol. 2011;22(8):1736-47.

10. Chaudary M, Millis R, Lane B, Miller NA. Paget's disease of the nipple: A ten year review including clinical, pathological, and immunohistochemical findings. Breast Cancer Res Treat. 1986;8(2):139-46.

11. Zheng S, Song QK, Zhao L, Huang R, Sun L, Li J, et al. Characteristics of mammary Paget's disease in China: a nationalwide multicenter retrospective study during 1999-2008. Asian Pac J Cancer Prev. 2012;13(5):1887-93.

12. Albarrán JM, Tavares A, Saldivia F, Prince J, Ramos S, Gutierrez N, et al. Enfermedad de Paget de la mama. Reporte de 13 casos. Rev Venez Oncol. 2010;22(3):194-200.

13. Freitas-Junior R, Siqueira LB, Carrijo ENA, Lacerda RP, Paulinelli RR, Rahal RM, et al. Variaçáo temporal do tratamento cirúrgico do câncer de mama em um hospital universitário na região CentroOeste do Brasil. Rev Col Bras Cir. 2013;40(3):180-85.

14. Rossi KKC. Doença de Paget de mama [Dissertação]. Campinas: Universidade Estadual de Campinas. Faculdade de Ciências Médicas; 2003.

15. Lim HS, Jeong SJ, Lee JS, Park MH, Kim JW, Shin SS, et al. Paget disease of the breast: mammographic, US, and MR imaging findings with pathologic correlation. Radiographics. 2011;31(7):1973-87.

16. Gabbi TVB, Valente NYS, Castro LGM. Doença de Paget pigmentada do mamilo simulando melanoma cutâneo: importância da imuno-histoquímica na diferenciação dessas doenças. An Bras Dermatol. 2006;81(5):457-60.

17. Hida T, Yoneta A, Nishizaka T, Ohmura T, Suzuki Y, Kameshima $\mathrm{H}$, et al. Pigmented mammary Paget's disease mimicking melanoma: report of three cases. Eur J Dermatol. 2012;22(1):121-4.

18. Marczyk E, Kruczak A, Ambicka A, Mularz K, Harazin-Lechowska A, Moskal J, et al. The routine immunohistochemical evaluation in Paget disease of the nipple. Pol J Pathol. 2011;62(4):229-35.

19. Sek P, Zawrocki A, Biernat W, Piekarski JH. HER2 molecular subtype is a dominant subtype of mammary Paget's cells. An immunohistochemical study. Histopathology. 2010;57(4):564-71.

20. Cirqueira MB, Moreira MAR, Soares LR, Freitas-Junior R. Subtipos moleculares do câncer de mama. Femina. 2011;39(10):499-503.

21. Swain SM, Kim SB, Cortés J, Ro J, Semiglazov V, Campone M, et al. Pertuzumab, trastuzumab, and docetaxel for HER2-positive metastatic breast cancer (CLEOPATRA study): overall survival results from a randomised, double-blind, placebo-controlled, phase 3 study. Lancet Oncol. 2013;14(6):461-71.

22. Coates AS, Winer EP, Goldhirsch A, Gelber RD, Gnant M, PiccartGebhart M, et al; Panel members. Tailoring therapies - improving the management of early breast cancer: St GallenInternational Expert Consensus on the Primary Therapy of Early Breast Cancer 2015. Ann Oncol. 2015 May 4. [Epub ahead of print]. pii: mdv221.

23. Coleman MP, Quaresma M, Berrino F, Lutz JM, De Angelis $\mathrm{R}$, Capocaccia $\mathrm{R}$, et al. Cancer survival in five continents: a worldwide population-based study (CONCORD). Lancet Oncol. 2008;9(8):730-56.

24. Nunes RD. Estudo de sobrevida das mulheres com câncer de mama na cidade de Goiânia, entre 1995 e 2003 [Dissertação]. Goiânia: Universidade Federal de Goiás. Faculdade de Medicina; 2011.

25. Li YJ, Huang XE, Zhou XD. Local Breast Cancer Recurrence after Mastectomy and Breast-Conserving Surgery for Paget's Disease: A Meta-Analysis. Breast Care (Basel). 2014;9(6):431-4. 
26. Caliskan M, Gatti G, Sosnovskikh I, Rotmensz N, Botteri E, Musmeci S, et al. Paget's disease of the breast: the experience of the European institute of oncology and review of the literature. Breast Cancer Research and Treatment. 2008;112(3):513-21.

27. Trebska-McGowan K, Terracina KP, Takabe K. Update on the surgical management of Paget's disease. Gland Surg. 2013;2(3):137-42.

28. Paulinelli RR, de Oliveira VM, Bagnoli F, Chade MC, Alves KL, FreitasJunior R. Oncoplastic mammaplasty with geometric compensation--a technique for breast conservation. J Surg Oncol. 2014;110(8):912-8.
29. Chagpar AB, Killelea BK, Tsangaris TN, Butler M, Stavris K, Li F, et al. A Randomized, Controlled Trial of Cavity Shave Margins in Breast Cancer. N Engl J Med. 2015 May 30. [Epub ahead of print]. DOI: 10.1056/NEJMoa1504473.

30. Allemani C, Weir HK, Carreira H, Harewood R, Spika D, Wang XS, et al.; the CONCORD Working Group. Global surveillance of cancer survival 1995-2009: analysis of individual data for 25676887 patients from 279 population-based registries in 67 countries (CONCORD-2). Lancet. 2015;385(9972):977-1010. 